\title{
La interactividad en las aplicaciones periodísticas para iPad italianas y españolas
}

\author{
Teresa Nozal CANTARERo \\ Universidade da Coruña \\ tnozal@udc.es \\ Ana GonZÁlez NeIRA \\ Universidade da Coruña \\ agonzalezn@udc.es
}

Recibido: 30/07/2012

Aceptado: 29/10/2012

\begin{abstract}
Sumario
Este trabajo analiza la interactividad de las aplicaciones para iPad de las cinco cabeceras de información general con mayor difusión en España e Italia. Para alcanzar el objetivo de la comunicación se estudia el grado de personalización, participación y servicios interactivos que ofrecen las aplicaciones de los periódicos objeto de estudio. Se tienen en cuenta las distintas versiones de cada cabecera que existen hoy en día en el Apple Store. Este trabajo se enmarca en un proyecto más amplio sobre convergencia de aplicaciones periodísticas en tableta llevado a cabo por el Grupo de Cultura y Comunicación Interactiva de la Universidade da Coruña.
\end{abstract}

Palabras clave: prensa, tabletas, interactividad, sistemas comparados, iPad

\section{Interactivity in Italian and Spanish newspapers iPad apps}

\begin{abstract}
This paper analyzes the interactivity in the iPad apps of the five largest general information newspapers in Spain and Italy. To achieve the goal of the paper we study the degree of personalization, participation and interactive services offered by regular apps under study. It takes into account the different versions of each header that exist today in the Apple Store. This work is part of a larger project on convergence journalism tablet applications conducted by Group Interactive Culture and Communication, University of A Coruña.
\end{abstract}

Keywords: press, tablet, interactivity, comparing media system, iPad

\section{Referencia normalizada}

NOZAL CANTARERO, Teresa y GONZÁLEZ NEIRA, Anas (2012): "La interactividad en las aplicaciones periodísticas para iPad italianas y españolas". Estudios sobre el mensaje periodístico. Vol. 18, núm. especial noviembre, págs.: 639-648. Madrid, Servicio de Publicaciones de la Universidad Complutense.

Sumario: 1. Introducción. 2. Metodología. 3. Desarrollo: resultados y discusión; 3.1. Interactividad; 3.1.1. Personalización; 3.1.2. Participación; 3.1.3. Servicios añadidos. 4. Conclusiones. 5. Referencias bibliográficas.

\section{Introducción}

Las empresas periodísticas han visto en las tabletas una oportunidad de negocio capaz de aligerar la carga de la crisis actual. Junto a su veloz difusión en el mercado, diversos informes destacan que la lectura de noticias se sitúa como segunda actividad más frecuente entre los usuarios de tabletas en España (AEDE, 2011).

Siguiendo el proceso de mediamorfosis (Fidler, 1997), la aparición de este nuevo soporte lleva consigo la redefinición de la identidad de las cabeceras periodísticas tanto en papel y web como en dispositivos móviles, hasta llegar a la diferenciación de 
los diarios en tableta frente a los otros canales. Actualmente se han identificado al menos tres tipos de contenidos ofrecidos por las cabeceras en el iPad:

1. La versión web del periódico, ya sea accesible directamente mediante navegador o mediante una app, adaptada y volcada de modo automático y en ocasiones específicamente diseñada para iPad y otras tabletas. En este trabajo se hace alusión a estas últimas denominándolas aplicaciones adaptadas.

2. El periódico en pdf diseñado y compaginado tal cual se imprime en papel, enriquecido con mayor o menor contenido interactivo y multimedia. Denominado en este artículo pdf enriquecido.

3. La versión específicamente creada para iPad, con contenidos y diseño diferentes del impreso y de la web. A este tipo de app se hace alusión denominándola nativa.

Este trabajo analiza las características de interactividad de las aplicaciones adaptadas, nativas y pdf enriquecido para iPad de las cinco cabeceras de información general con mayor difusión en España e Italia, países pertenecientes al paradigma pluralista polarizado siguiendo la clasificación establecida por Daniel Hallin y Paolo Mancini (2008). Según los datos de la OJD y Audipress de 2012, los diarios españoles e italianos con mayor difusión son El País, El Mundo, ABC, La Vanguardia y El Periódico y los italianos Corriere della Sera, Repubblica, La Stampa, Il Messaggero y Il Resto del Carlino.

Los periódicos españoles, excluyendo El Periódico de Catalunya, han optado por dos versiones de la cabecera, una en pdf más o menos enriquecido, y otra con un diseño adaptado y contenidos volcados de la web. Han incorporado al iPad sus dos ediciones preexistentes, la de papel y la web, para no perder ningún público objetivo. De momento no hay en España aplicaciones nativas entendidas como han sido descritas anteriormente. En Italia, solo tres de las cinco cabeceras poseen otra versión además del pdf, en este caso nativas y no existen versiones adaptadas de la web. Repubblica merece una mención especial ya que su app nativa es una edición vespertina, $R$ Sera. Parece ser una buena oportunidad de negocio puesto que los estudios de consumo de prensa en el iPad manifiestan un índice de lectura mayor durante la noche (Macri 2011, Faas 2012).

Los pdf enriquecidos de España se comercializan mayoritariamente a través de dos grandes quioscos: Orbyt y Kiosko y más. En Italia no existe ningún tipo de iniciativa similar.

La literatura científica sobre prensa en iPad o tabletas en general es escasa ya que los tiempos académicos son más lentos que los profesionales y del mercado. Por eso, muchas de las publicaciones sobre tabletas proceden de consultoras (Accenture, Price Waterhouse Coopers), asociaciones del ramo (AIMIC, Ametic, AEDE) y laboratorios de investigación. Dichas publicaciones están centradas mayoritariamente en el funcionamiento de la tableta, usabilidad, perfil de usuario y difusión comercial.

Dentro del mundo académico existen varias tesis (Cobos 2011, Benckert van de Boel 2011, Da Cunha 2011, Haeger 2011) que abordan de un modo más concreto aspectos de la prensa en el iPad. También se han presentado comunicaciones en congresos como los celebrados por ECREA, o en citas anuales como el International 
Symposium on Online Journalism de Austin (Texas) o el de Periodismo Digital de Huesca. No obstante, hasta el momento los únicos libros editados sobre periódicos en el iPad han sido Dalle gazzette all'iPad de Elena Valentini (2012), con un valioso corpus teórico, y el escrito por Mario García The iPad design Lab (2012), con interesantes aportaciones para diseñadores. Además cabe citar las aportaciones de Edo (2011), Cabrera González y Bernal Triviño (2011) sobre las posibilidades que las tabletas ofrecen al periodismo.

Por último, dado que en esta investigación se analiza el grado de interactividad de las periódicos digitales, aunque centrado en apps para iPad, cabe destacar los trabajos publicados al respecto por la profesora Cabrera González (2010), Martínez Martínez (2008), Masip, Díaz Noci, Domingo, Micó Sanz y Salaverría (2010) y el coordinado por Marcos Palacios (2011) acerca de herramientas de evaluación de la calidad, todos sobre ciberperiodismo.

\section{Metodología}

La metodología llevada a cabo en esta investigación comienza con el diseño de unas fichas de análisis. Para ello se selecciona una muestra de 75 versiones de periódicos ofrecidos a través de la Apple Store que puedan ser categorizadas como aplicaciones periodística frente a las que son primordialmente informativas y a las de infoTabment ${ }^{1}$. A continuación se revisa en paralelo para buscar elementos susceptibles de convertirse en ítems de análisis en función de tres parámetros: usabilidad, interactividad y contenidos multimedia. Asimismo se valora la función periodística de los contenidos multimedia y de la compaginación teniendo en cuenta en este último caso las versiones papel y web de la cabecera.

Tras este proceso se obtiene una primera versión de la tabla con 279 ítems cuya codificación se somete a testeo por dos investigadores expertos ajenos a este trabajo. Tras dicho testeo varios ítems son recodificados y otros eliminados hasta originar una tabla de 239 elementos.

Tras la observación los investigadores se plantean las siguientes hipótesis en lo relativo a interactividad:

1. Las cabeceras en el iPad mantienen características de interactividad de la web respecto al grado de personalización ${ }^{1}$.

2. Las cabeceras en el iPad mantienen características de interactividad de la web respecto al grado de participación ${ }^{2}$.

1 El concepto infoTabment surge de la aplicación del infotainment a la nueva actitud detectada entre los usuarios de tableta cuando consumen información y entretenimiento. Fue utilizado por primera vez en las investigaciones realizadas en 2011 por el Grupo de Investigación Cultura y Comunicación Interactiva de la Universidade da Coruña sobre el comportamiento de lectores de prensa en iPad. Para una definición más precisa de los tres criterios, véase Sanjuán, Nozal y González 2012.

2 Se entiende por personalización la posibilidad que tiene el usuario de adaptar cualquier característica de la aplicación a sus necesidades o intereses.

3 Se entiende por participación la posibilidad que tiene el usuario de interactuar con los contenidos periodísticos ya sea comentándolos, compartiéndolos o incorporando otros nuevos. 
3. Las cabeceras en el iPad mantienen características de interactividad de la web respecto a los servicios añadidos ${ }^{3}$ ofrecidos.

Para responder a las hipótesis planteadas, la tabla de 239 ítems vuelve a someterse a un testeo para seleccionar los elementos relativos al objeto de esta investigación. Se obtiene una tabla definitiva de 27 elementos que se aplica a las versiones de iPad de los diez diarios españoles e italianos en dos días distintos de la semana entre los meses de abril a junio del 2012. En meses posteriores se revisan y corregen los datos en función de la evolución de las aplicaciones. Del vaciado de contenidos y la confrontación de datos se obtienen los resultados que se exponen a continuación. Los ítems relativos a cada hipótesis pueden leerse en las tablas que acompañan los gráficos.

\section{Desarrollo: resultados y discusión}

\subsection{Interactividad}

A continuación se exponen los aspectos destacables relativos a personalización, participación y servicios interactivos, rasgos con los que se evalúa el nivel de interactividad.

\subsubsection{Personalización}

\section{Personalización comparada}

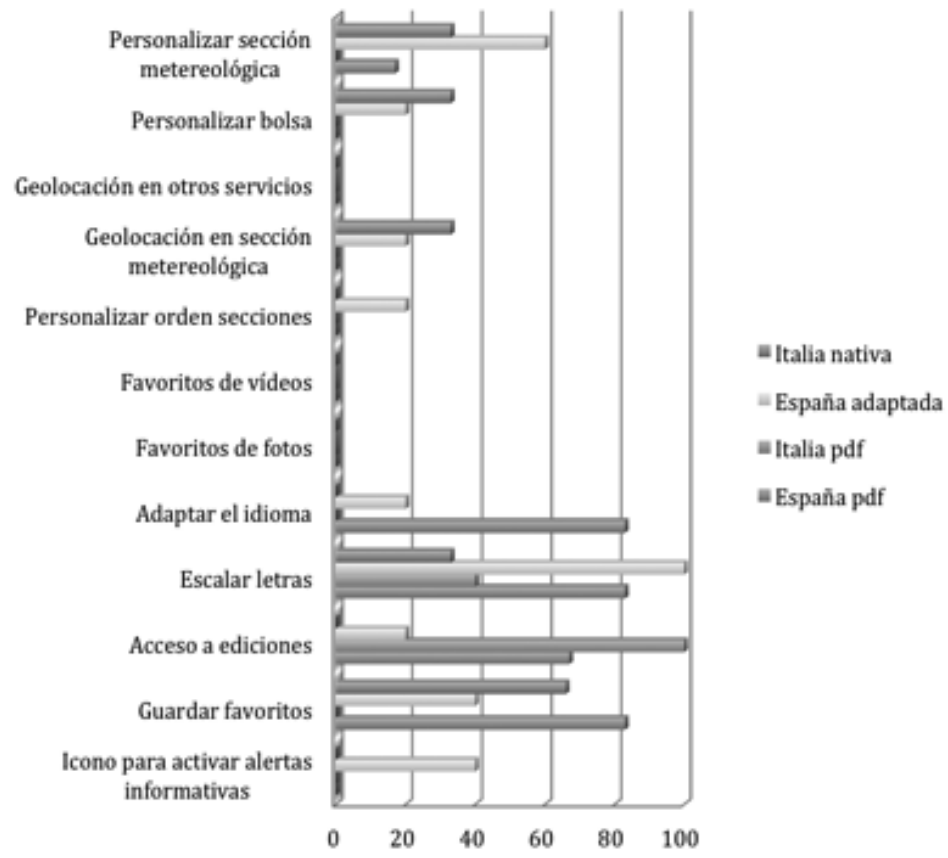

${ }^{4}$ Se entiende por servicios añadidos interactivos cualquier enriquecimiento en secciones tradicionales no estrictamente periodísticas (cartelera, esquelas, bolsa, pasatiempos, etc.) y otros más vinculados a la web como buscadores de información o interactividad en infografías. 


\begin{tabular}{|l|c|c|c|c|}
\hline \multicolumn{4}{|c|}{ Personalización comparada } \\
\hline & $\begin{array}{c}\text { España } \\
\text { pdf }\end{array}$ & $\begin{array}{c}\text { Italia } \\
\text { pdf }\end{array}$ & $\begin{array}{c}\text { España } \\
\text { adaptada }\end{array}$ & $\begin{array}{c}\text { Italia } \\
\text { nativa }\end{array}$ \\
\hline Icono para activar alertas informativas & 0 & 0 & 40 & 0 \\
\hline Guardar favoritos & 83 & 0 & 40 & 66 \\
\hline Acceso a ediciones & 67 & 100 & 20 & 0 \\
\hline Escalar letras & 83 & 40 & 100 & 33 \\
\hline Adaptar el idioma & 83 & 0 & 20 & 0 \\
\hline Favoritos de fotos & 0 & 0 & 0 & 0 \\
\hline Favoritos de vídeos & 0 & 0 & 0 & 0 \\
\hline Personalizar orden secciones & 0 & 0 & 20 & 0 \\
\hline Geolocación en sección metereológica & 0 & 0 & 20 & 33 \\
\hline Geolocación en otros servicios & 0 & 0 & 0 & 0 \\
\hline Personalizar bolsa & 0 & 0 & 20 & 33 \\
\hline Personalizar sección metereológica & 17 & 0 & 60 & 33 \\
\hline
\end{tabular}

Tabla 1. Elementos de personalización de las versiones. Elaboración propia.

Según los datos analizados las cabeceras españolas ofrecen más posibilidades de personalización y adaptación a los gustos o necesidades del lector que las italianas. Sin embargo, en ciertos casos este principio no se cumple de modo claro. Por ejemplo, mientras que todos los pdf italianos incluyen la opción de acceso a ediciones, tan sólo el $67 \%$ de los españoles lo facilita en su pdf. El equilibrio se recupera al observar las versiones nativas italianas y adaptadas españolas, porque ninguna italiana da opción a ediciones mientras que una de las españolas sí lo permite (La Vanguardia).

Algo parecido sucede con el icono de guardar favoritos. Aunque los diarios españoles lo proporcionan, puesto que el $83 \%$ de sus pdf lo hace frente a ninguno de los italianos, en las versiones adaptadas y nativas este dato se iguala, dado que en ambos países dos cabeceras lo ofrecen. No obstante, no siempre queda claro dónde se almacenan estas informaciones favoritas ya que en algunos de los periódicos analizados el usuario desconoce el lugar desde el cual puede recuperar la información.

Como ya se ha mencionado, los diarios españoles ofrecen más opciones de personalización. Así sucede en la posibilidad de escalar letras. En este sentido, destaca el servicio Smart Flow que Kiosko y más facilita, en el que se puede elegir entre un número limitado de tipografías.

En las ediciones adaptadas y nativas se permite personalizar la sección de meteorología, si bien el número de cabeceras con dicha opción es mayor en España. Especial atención merece El Mundo en su versión adaptada, único periódico que consiente personalizar el orden de las secciones.

Respecto a la selección del idioma, ninguna de las adaptadas y nativas lo permite, exceptuando el caso de El Periódico, lo cual resulta llamativo teniendo en cuenta que algunas regiones italianas y autonomías españolas son bilingües. Esta característica varía en el caso de los pdf españoles, ya que los ofrecidos por Kiosko y más posibilitan la selección entre una lista de 14 idiomas, ninguno autonómico, mientras que los italianos no lo facilitan en ningún caso. 
A pesar de que la actualización y la información de última hora sea una de las ventajas que puede incorporar el iPad, el icono de alertas informativas existe solamente en dos de los diarios españoles y tan sólo en sus versiones adaptadas. En ningún caso los italianos ofrecen esta posibilidad. Un periódico como El Pais, que facilita esta opción en su web y en la versión adaptada del iPad, no la incorpora en la versión en pdf, a pesar del atractivo añadido que podría suponer para un lector que accede al periódico a través de Kiosko y más. Podría entenderse que las notificaciones que la aplicación solicita poder enviar al ser instalada sustituyen el servicio que ofrece el icono de alertas informativas, si bien no es exactamente lo mismo. Además, en el caso de que no se dé el permiso de notificaciones, la aplicación no da opción a configurar alertas informativas.

\subsubsection{Participación}

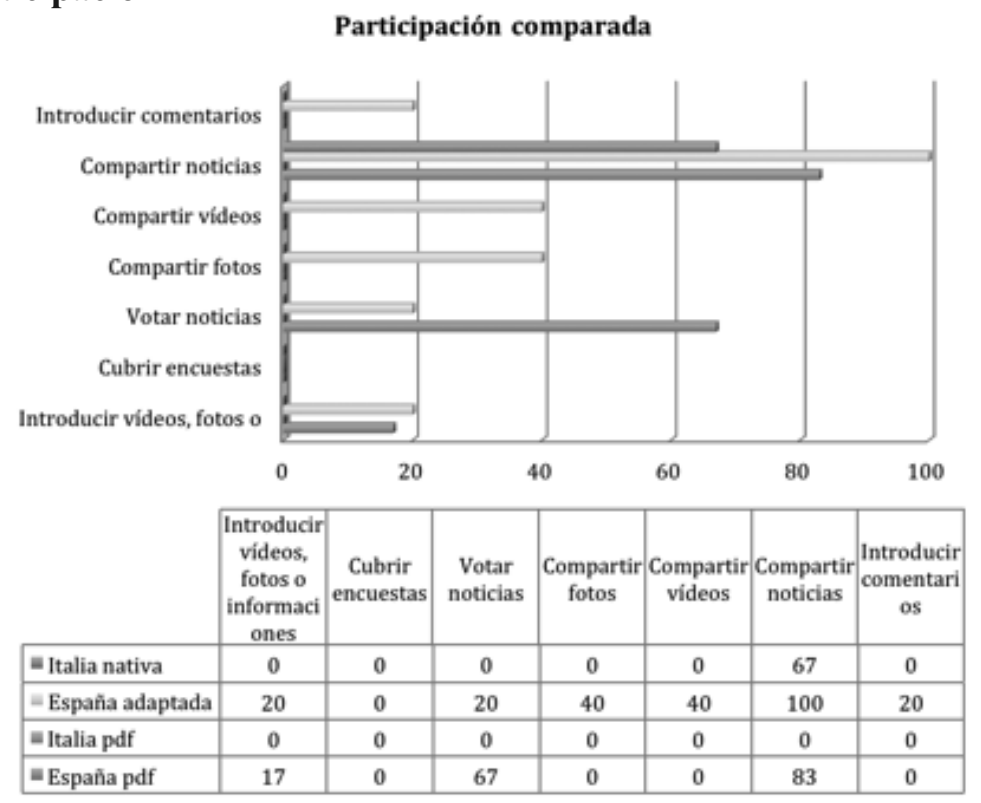

Tabla 2. Elementos de participación presentes en las versiones. Elaboración propia.

La interacción con el medio convierte al iPad en un soporte muy atractivo para la cabecera, pues puede aportar un alto grado de información sobre los usuarios (dejando a un lado los acuerdos comerciales con las plataformas de venta como Apple Store). Los resultados obtenidos demuestran que los periódicos españoles ofrecen muchas más opciones de participación que los italianos, y aún así son escasas. En los demás casos tal interacción se limita a la opción de votar las noticias, que se confirma en el $67 \%$ de los pdf españoles y el $20 \%$ de las versiones adaptadas, frente al $0 \%$ de los italianos tanto en pdf como nativas.

El icono de compartir noticias (a través de correos electrónicos o redes sociales) se generaliza en el caso de las cabeceras españolas con el 100\% de las versiones adaptadas y el $83 \%$ de los pdf. En Italia, solo el $67 \%$ de las versiones nativas ofrecen esta posibilidad mientras que ningún pdf lo contempla. 
Por lo que respecta a la posibilidad de compartir fotos o vídeos, de nuevo solo dos diarios españoles - El País y El Mundo en el caso de las fotos y El País y La Vanguardia en el de los vídeos- incluyen esta opción en sus versiones adaptadas frente a ninguna italiana. Este rasgo pone de manifiesto la importancia de la información como elemento social en España, como producto de consumo en grupo y no exclusivamente individual (Cabrera, 2010, Valentini, 2012).

A excepción de El Mundo en su versión pdf, ninguno de los diarios analizados permite cubrir encuestas. Esta cabecera también posibilita enviar cartas al director a través de su pdf. Tan sólo uno, La Vanguardia, posibilita la opción de introducir vídeos, fotos, comentarios e informaciones.

\subsubsection{Servicios añadidos}

\section{Servicios interactivos}
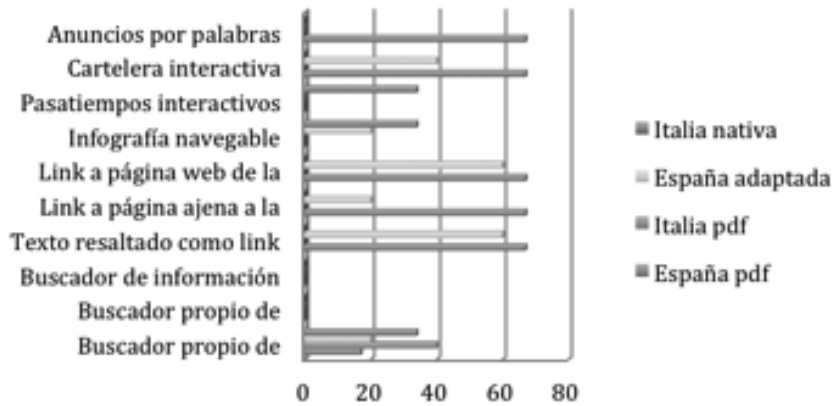

\begin{tabular}{|l|c|c|c|c|}
\hline \multicolumn{5}{|c|}{ Servicios interactivos } \\
\hline & España pdf & Italia pdf & $\begin{array}{c}\text { España } \\
\text { adaptada }\end{array}$ & $\begin{array}{c}\text { Italia } \\
\text { nativa }\end{array}$ \\
\hline $\begin{array}{l}\text { Buscador propio de información dentro del pe- } \\
\text { riódico }\end{array}$ & 17 & 40 & 20 & 34 \\
\hline $\begin{array}{l}\text { Buscador propio de información fuera del pe- } \\
\text { riódico }\end{array}$ & 0 & 0 & 0 & 0 \\
\hline Buscador de información externo & 0 & 0 & 0 & 0 \\
\hline Texto resaltado como link & 67 & 0 & 60 & 0 \\
\hline Link a página ajena a la cabecera & 67 & 0 & 20 & 0 \\
\hline Link a página web de la cabecera & 67 & 0 & 60 & 0 \\
\hline Infografía navegable & 0 & 0 & 20 & 34 \\
\hline Pasatiempos interactivos & 0 & 0 & 0 & 34 \\
\hline Cartelera interactiva & 67 & 0 & 40 & 0 \\
\hline Anuncios por palabras interactivos & 67 & 0 & 0 & 0 \\
\hline
\end{tabular}

Tabla 3. Servicios interactivos presentes en las versiones. Elaboración propia.

En general, las cabeceras españolas ofrecen más servicios interactivos que las italianas. No obstante algún servicio, como los pasatiempos interactivos, sólo existe en un periódico italiano, Corriere della Sera en su versión nativa, mientras que en España 
ninguno contempla dicha opción. Italia también tiene mayor oferta en buscadores de información propios que lanzan búsquedas dentro del periódico: el $40 \%$ de las versiones en pdf de los diarios italianos frente al $17 \%$ de los españoles.

En el resto de los ítems que sirven para evaluar el grado de servicios interactivos vuelve a imponerse una mayor oferta española. Así, el 67\% de los pdf españoles y el $60 \%$ de las versiones adaptadas ofrecen texto resaltado como link que enlaza con páginas del periódico. Además todos los pdf y el $20 \%$ de los diarios españoles adaptados en algunos de esos enlaces conecta con páginas externas a la cabecera. En el caso de los españoles en pdf esta característica se debe a la aplicación de Kiosko y más que convierte automáticamente cualquier dirección url o correo electrónico en un enlace. Por consiguiente, tanto la sección de cartelera como los anuncios por palabras permiten acceder a las webs de cines, servicios de compras y cualquier otra página cuya dirección url esté escrita. Destaca la ausencia de hipervínculos en los periódicos italianos analizados, por lo que no se explota el enriquecimiento de la información. Esta escasez de hipervínculos se explica porque los editores consideran que pueden interrumpir y dificultar la navegación en su diario.

\section{Conclusiones}

En relación a las hipótesis planteadas y a tenor de los resultados obtenidos tras el análisis de los ítems de estudio se confirma que:

La hipótesis 1 "Las cabeceras en el iPad mantienen características de interactividad de la web respecto al grado de personalización". No se acepta. Ni las cabeceras españolas ni las italianas accesibles a través de apps en el iPad ofrecen las mismas características de personalización que la web, ni siquiera en el caso de las adaptadas con volcado casi automático de contenidos.

La hipótesis 2 "Las cabeceras en el iPad mantienen características de interactividad de la web respecto al grado de participación". No se acepta. En ningún caso se llega al nivel de participación que ofrece la web ni en compartir contenidos en redes sociales o por correo, ni en interactuar con el medio, ni en modificar o incorporar nuevos contenidos.

La hipótesis 3 "Las cabeceras en el iPad mantienen características de interactividad de la web respecto a los servicios añadidos ofrecidos". No se acepta. Ni en España ni en Italia se llega a ofrecer en las apps de los periódicos analizados el mismo tipo, cantidad y nivel de especialización de servicios interactivos que se proporciona en la web.

Esta investigación pone de manifiesto que las aplicaciones de periódicos en el iPad se encuentran todavía en un estadio inicial de su desarrollo. La adaptación, sobre todo en el caso español, bebe de las experiencias en papel y web de las distintas cabeceras y apenas incorpora elementos de interactividad que puedan mejorar la calidad de lectura del público en el medio específico de la tableta. Una mayor inversión en el desarrollo de la interactividad, diseño y otras características de estas aplicaciones podría abrir nuevos públicos objetivos que ofrecieran posibilidad de negocio, en vez de arrastrar los ya preexistentes de papel y web.

La comparación de las aplicaciones de ambos países pone de manifiesto que los diarios italianos son más conservadores (apenas incorporan elementos de participación o interactividad) frente a los españoles. Sin embargo, en Italia encontramos las únicas 
versiones de diarios creadas exclusivamente para tabletas independientes de sus versiones en papel y web, La Stampa top news, Corriere ipad y RSera, manifestación de una apuesta mayor por este dispositivo móvil en algunos diarios. Resulta evidente que las estrategias de incorporación de cabeceras al iPad se homogeneizan en el caso de España pero no en Italia. Es posible que la homogeneización de estrategias en España se deba precisamente al hecho de que existan los quioscos en los que se ofrecen los pdf enriquecidos de casi todas las cabeceras, mientras que las versiones adaptadas de la web se proporcionan de manera independiente. Como se ha apuntado en la introducción, los quioscos no existen en Italia, por lo que las alianzas que suponen y que supuestamente rentabilizan las acciones al unificar esfuerzos quedan vedadas a las cabeceras de este país.

En definitiva, es probable que en los próximos meses las cabeceras españolas desarrollen aplicaciones nativas en un intento de ir más allá de las volcadas del papel y la web que ofrecen en este momento. Según se puede deducir de los resultados de esta investigación, las características de interactividad que incorporarán serán mayores en cantidad y calidad que las nativas italianas actuales.

\section{Referencias bibliográficas}

ACCENTURE (2011): Retos y oportunidades del universo digital móvil en España: más ubicuo, más social, más personal. Informe 2011. Madrid, Accenture.

AIMIC (2011): La prensa: digital vs papel. http://www.aimc.es/spip.php?action=acceder_document\&arg $=1817 \& \mathrm{cle}=301 \mathrm{c} 9291$ dbedaaa2e 29 aa35d8f6ef681\&file $=$ pdf \%2Fimm_prensa2011.pdf [fecha de consulta: 15 de junio de 2012]

AMETIC (2011): Informe 2011 de la Industria de Contenidos Digitales. Asturias, Ametic.

BENCKERT VAN DE BOEL, Anna (2011): Designing the future of the newspaper. Tesis de master presentada en la Universidad de Malmö.

CABRERA GONZÁLEZ, Ma Ángeles (2010): "La interactividad de las audiencias en entornos de convergencia digital". Icono14, $\mathrm{n}^{\circ} 15$. http://www.icono14.net/Num.-15.-Interactividad/la-interactividad-de-audiencias. [fecha de consulta: 20 de julio de 2012]

CABRERA GONZÁLEZ, María Ángeles y BERNAL TRIVIÑO, Ana Isabel (2011): "Tabletas, smartphones, medios online, contenidos y consumos", en Actas del $I$ Congreso de Comunicación y Educación. Estrategias de alfabetización mediática: reflexiones sobre comunicación y educación. Barcelona, Universidad Autónoma de Barcelona.

COBOS, Tania Lucía (2011): Periodismo móvil: la prensa mexicana y su incursión en plataformas móviles. Casos El Universal y El Norte. Monterrey, México, Tesis de maestría presentada en el Tecnológico de Monterrey.

CUNHA, Rodrigo do Espírito Santo da (2011): Revistas no cenário da mobilidade: a interface das edições digitais para Tablets. Dissertação apresentada ao Programa de Pós-graduação em Comunicação e Cultura Contemporâneas da Universidade Federal da Bahia, como requisito parcial para a obtenção do título de Mestre. Uni- 
versidade Federal da Bahia. Faculdade de Comunicação. Programa de Pos-graduaçao em comunicação e cultura contempoâneas. Salvador, doc. en pdf: https://repositorio.ufba.br/ri/bitstream/ri/5959/1/Rodrigo\%20Cunha.pdf

DIAZ NOCI, Javier (2008): "Definición teórica de las características del ciberperiodismo: elementos de comunicación digital". Doxa Comunicación, $n^{\circ}$ 6, pp. 53-91.

EDO, Concha (2011): "Las tablets no son la panacea para la prensa pero pueden facilitar su evolución", en Libro Blanco de la Prensa Diaria 2012. Madrid, AEDE.

FIDLER, Robert (1997): Mediamorfosis: Comprender los nuevos medios. Buenos Aires, Granica.

GARCÍA, Mario (2012): iPad design lab. Libro electrónico, iTunes

HAEGER, Anna (2011): Publishing Solutions for Tablets and E-Readers. Tesis de máster. Stockholm, Royal Institute of Technology.

MACRI, Piero (2011): Il potenziale di Smartphone e tablet. http://it.ejo.ch/4954/nuovi -media/il-potenziale-di-smartphone-e-tablet [fecha de consulta 20 de julio de 2012]

MARTÍNEZ MARTÍNEZ, Silvia (2008): "La interactividad en los diarios digitales españoles”. Doxa Comunicación, n $^{\circ}$ 7, pp. 157-164.

MASIP, Pere, DÍAZ NOCI, Javier, DOMINGO, David, MICÓ SANZ, Josep Luís, SALAVERRIA, Ramón (2010): "Investigación internacional sobre ciberperiodismo: hipertexto, interactividad, multimedia y convergencia". El Profesional de la Información, $\mathrm{n}^{\circ} 19$ (6), noviembre-diciembre, pp. 568-576.

PALACIOS, Marcos (coord.) (2011): Ferramentas para a analise de Qualidade no ciberjornalismo. Covilha, Labcom books.

PWC (2011): Global entertainment and media outlook 2011-2015. http://boletines.prisadigital.com/PwCOutlook2011-Industry\%20overview.pdf. [fecha de consulta: 13 de julio de 2012].

VALENTINI, Elena (2012): Dalle gazzette all'iPad. Il giornalismo al tempo dei tablete. Milano, Mondadori.

\section{Teresa NOZAL CANTARERO}

Contratada doctora. Facultad de Comunicación. Universidade da Coruña. Miembro del Grupo de Investigación de Cultura y Comunicación Interactiva. Correo electrónico: tnozal@udc.es

\section{Ana GONZÁLEZ NEIRA}

Ayudante doctora. Facultad de Comunicación. Universidade da Coruña. Miembro del Grupo de Investigación de Cultura y Comunicación Interactiva Correo electrónico: agonzalezn@udc.es

Dirección postal de las autoras

Facultad de Comunicación

Campus de Elviña

Universidade da Coruña

15071 A Coruña 